\title{
A NOTE ON TWO-SIDED IDEALS IN $C^{*}$-ALGEBRAS ${ }^{1}$
}

\author{
JOHN BUNCE
}

Abstract. An elementary proof is given of the fact that $(I+J)^{+}$ $=I^{+}+J^{+}$for $I$ and $J$ closed two-sided ideals in a $C^{*}$-algebra.

In this note we give a short and elementary proof of the following theorem, which was proposed as a problem by J. Dixmier [1, Problem 1.9.12] and first proved by E. St $\phi$ rmer [4].

THEOREM. Let $A$ be a $C^{*}$-algebra. If I and $J$ are uniformly closed two-sided ideals in $A$ then $(I+J)^{+}=I^{+}+J^{+}$.

Here $I^{+}$denotes the set of positive elements in $I$. To prove the theorem we may assume that $A$ has an identity, denoted by $e$. In [4] Størmer proved the theorem by using some results of E. G. Effros [2] and some quite delicate calculations using the functional calculus to prove the following lemma, from which the theorem follows by a straightforward induction argument.

Lemma. With the assumptions as in the theorem, let a belong to $(I+J)^{+}$, and let $\epsilon>0$ be given. Then there exists $b$ in $I^{+}$and $c$ in $J^{+}$such that $0 \leqq a-b-c \leqq \epsilon e$.

A SHORT PROOF OF THE LEMMA. Let $a$ belong to $(I+J)^{+}$. Then $a=f+g$ for some $f$ in $I$ and $g$ in $J$. Since $a=a^{*}$, we may assume that $f=f^{*}$ and $g=g^{*}$. Let $h=|f|+|g|+\epsilon e$. Then $h$ is invertible and 0 $\leqq a \leqq h$. Let $d=a^{1 / 2} h^{-1 / 2}$. Then $d$ is in $A$, and $0 \leqq d^{*} d=h^{-1 / 2} a h^{-1 / 2}$ $\leqq h^{-1 / 2} h h^{-1 / 2}=e$. Thus $d d^{*} \leqq e$, and $0 \leqq a-d|f| d^{*}-d|g| d^{*}=\epsilon d d^{*} \leqq \epsilon e$. Since $d|f| d^{*}$ is in $I^{+}$and $d|g| d^{*}$ is in $J^{+}$the lemma is proved.

We note that G. K. Pedersen has given another proof of this theorem [3].

\section{REFERENCES}

1. J. Dixmier, Les $C^{*}$-algèbres et leurs représentations, Cahiers Scientifiques, fasc. 29, Gauthier-Villars, Paris, 1964. MR 30 \#1404. 391-412.

2. E. G. Effros, Order ideals in a $C^{*}$-algebra and its dual, Duke Math. J. 30 (1963),

3. G. K. Pedersen, $A$ decomposition theorem for $C^{*}$-algebras, Math. Scand. 22 (1968), 266-268.

4. E. Stфrmer, Two-sided ideals in $C^{*}$-algebras, Bull. Amer. Math. Soc. 73 (1967), 254-257. MR 34 \#8210.

UNIVERSITY OF KANSAS, LAWRENCE, KANSAS 66044

Received by the editors May 11, 1970 and, in revised form, June 10, 1970.

AMS 1969 subject classifications. Primary 4665; Secondary 4660.

1 This was written while the author held an AFOSR postdoctorate. 